\title{
Intermediate evolution using SNIa, and BAO
}

\author{
Víctor H. Cárdenas and O. Herrera
}

\begin{abstract}
We study the intermediate evolution model and show that, compared with the recent study of a power-law evolution, the intermediate evolution is a better description of the low-redshift regime supported by observations from type Ia supernovae and BAO. We found also that recent data suggest that the intermediate evolution is as good a fit to this redshift range as the $\Lambda$ CDM model.
\end{abstract}

Keywords cosmology; dark energy

\section{Introduction}

The current cosmological paradigm was set primarily after discovering that type Ia supernovae (SNIa), a well-studied standard candle, are dimmer than expected in the context of the standard cosmological model (Riess et al. (1998); Perlmutter et al. (1999)). The simplest way to describe this observation in the context of the standard big-bang picture is by introducing a cosmological constant term, $\Lambda$, into Einstein's field equations.

In this setup the cosmological constant $\Lambda$ drives the current accelerated expansion of the universe, leading to the successful $\Lambda \mathrm{CDM}$ model, the simplest one that fits a varied set of observational data such as SNIa, measurements of the baryon acoustic oscillations (BAO), information from the cosmic microwave background radiation (CMBR), growth of structure, etc (Weinberg et al. (2013); Perivolaropoulos (2010)).

However, this model looks unnatural in at least two ways; (i) there is no clue about the physical mechanism to get the current value for $\Omega_{\Lambda}$, and (ii) this model

Víctor H. Cárdenas and O. Herrera

Instituto de Física y Astronomía, Facultad de Ciencias, Universidad de Valparaíso, Av. Gran Bretaña 1111, Valparaíso, Chile tells us we live in a very special epoch where the cosmological constant contribution $\Omega_{\Lambda}$ is of the same order of magnitude as the dark matter contribution $\Omega_{M}$, a highly improbable fact considering the dark matter contribution decreases as $a^{-3}$, with $a(t)$ the scale factor. Meanwhile the cosmological constant contribution is and always has been a constant.

A plausible alternative is to explore deviations from the $\Lambda$ CDM model, assuming a variable $\Lambda$. Dark energy (DE) is the name of the unknown component responsible for the current accelerated expansion of the universe (Frieman et al. 2008). In its simplest form, this can be described by a fluid with constant equation of state (EoS) parameter $w=-1$ corresponding to a cosmological constant. There are also models where a scalar field drives the cosmic acceleration, so-called quintessence models (Martin 2008), as well as models where through a modification of the gravity sector the cosmic acceleration is described (Tsuijikawa 2010).

This honest approach focuses only on problem (ii), mentioned in the previous paragraph, but it is almost completely disconnected from problem (i). Most of the work in cosmology since the discovery of the accelerated expansion of the universe has been of this type. The efforts are focused on characterizing this new component, dark energy (DE), somehow disconnected from first principles.

Many scientists think that a real understanding of the DE problem, focusing this time on problem (i), i.e., the old well-known cosmological constant (C.C.) problem Weinberg 1989; Peebles and Ratra 2003), would certainly reveal a door to new physics.

Among the many attempts to tackle the cosmological constant problem, one of the most interesting is where a suitable relaxation mechanism drives the C.C. to a small value. For example, Dolgov (1997) describes a model where a scalar field is coupled to the curvature of the space-time, such that their contribution to the en- 
ergy density cancels the vacuum energy. Similar to this work are those proposed by Hebecker and Wetterich (2000), where an additional scalar field is introduced, which to date remains a C.C. which asymptotically vanishes (see Padmanabhan (2003) for a review).

In a recent paper (Dolgov et al. 2014) the authors used a power-law evolution $a(t) \simeq t^{\beta}$ - suggested by modified gravity theories at low redshift - and directly studied the constraints the supernova data imposed on the parameter $\beta$. They found, surprisingly, using both data from the Union 2.1 (Suzuki et al. 2012) and the recently released joint light-curve analysis (JLA) set (Betoule et al. 2014), that SNIa data suggest a $\beta \simeq 3 / 2$ assuming a flat universe. Although successful in producing a good fit to the SNIa data, the model is unable to describe a transition from a decelerated to an accelerated phase. In fact, by using a scale factor $a(t) \simeq t^{\beta}$, this implies a constant deceleration parameter $q=(1-\beta) / \beta$. This transition is a key ingredient that any model must satisfy if it claims to describe the recent (low redshift $z<1$ ) evolution of the universe, which is essentially the same redshift range as the supernova data span.

It is the purpose of this paper to explore a slightly different scale factor evolution - the so-called intermediate evolution - that interpolates between a power-law evolution and an exponential one, that is able to describe the transition from the decelerated phase to an accelerated one. We use the latest data sets of SNIa (some of which was used in (Dolgov et al. 2014)), and compare our results.

In the next section we introduce the intermediate evolution. In section III we describe the results of our study and the comparison with previous works. We end with a discussion section.

\section{The intermediate evolution}

The intermediate evolution was introduced first in the context of inflationary models as an exact solution for a scalar field potential of the type $V(\phi) \propto \phi^{-4\left(f^{-1}-1\right)}$ (Barrow 1990; Barrow \& Saich 1990; Muslinov 1990), where $f$ is a free parameter with range $0<f<1$. A potential of this form, in the context of the slow-roll approximation, gives a Harrison-Zel'dovich spectrum of density perturbations with an exact scale-invariant spectral index i.e., $n_{s}=1$.

The motivation to study an intermediate inflationary model comes from string/M theory. This theory suggests that in order to have a ghost-free action, high order curvature invariant corrections to the Einstein-Hilbert action must be proportional to the Gauss-Bonnet (GB) term (Boulware \& Deser 1985, 1986). GB terms arise naturally as the leading order of the expansion to the low-energy string effective action (Koivisto \& Mota 2007a b). This kind of theory has been applied to a possible resolution of the initial singularity problem (Antoniadis et al. 1994), to the study of Black-Hole solutions (Mignemi \& Stewart 1993; Kanti et al. 1996; Chen et al. 2007), and accelerated cosmological solutions (Noiiri \& Odintsov 2005; Noiiri et al. 2005; Cognola et al. 2006; Barrow et al. 2006; del Campo 2014), among others.

Particularly interesting to this work is the finding that, using a GB interaction with a scalar field $\phi$, it is possible to describe a DE model leading to a solution of the form $a(t)=a_{0} \exp \left[(2 /(\kappa n)) t^{1 / 2}\right]$ (Sanyal 2007, 2009). Here, $\kappa=8 \pi G$ and $n$ is an arbitrary constant. Actually, this is exactly a particular case of the intermediate evolution, in which the scale factor evolves as

$a(t)=b \exp \left(A t^{f}\right)$,

where $A$ is a positive constant and $f$ was introduced above. Thus, the expansion of the universe is slower than standard de Sitter inflation $(a(t)=\exp (H t))$, but faster than power law inflation $\left(a(t)=t^{p} ; p>1\right)$.

Using (1) the Hubble function is

$H=\frac{\dot{a}}{a}=A f t^{f-1}$,

and the deceleration parameter is

$q=-\frac{\ddot{a} a}{\dot{a}^{2}}=-1+\frac{1-f}{f} \frac{1}{A t^{f}}$.

This last result shows immediately that this kind of evolution makes it possible to describe the transition from a decelerated to an accelerated expansion phase. In fact, for small $t$ the second term dominates, which for $f<1$ gives a decelerating universe evolution, and for late (larger) times, the first factor starts to dominate, leading to an accelerated evolution.

From a purely theoretical point of view, most of the efforts made using the GB scenario to tackle the DE problem, have focused on obtaining solutions which look similar to the $\Lambda$ CDM model at the background level (see (Granda \& Jimenez 2014) and references therein). A very interesting result was found in (De Felice et al. 2010), where by studying the so-called general GB gravity, the authors show in the linear perturbations regime that there is an instability during the radiation and matter domination epoch. This modified GB gravity is equivalent to the term GB coupled to a scalar field, but without a kinetic term. There remains the uncertainty as to whether this instability appears 
once we turn on the kinetic term, as is the case of the model from which the intermediate evolution emerged. Although this instability does not spoil out the power of the model to describe DE, as the authors mentioned in the text, it is clear that this issue must be delved into more deeply and also in a more general setup (see for example de la Cruz-Dombriz \& Saez-Gomez (2012)).

More interesting for the present work are the results from a direct test against observational data. In Koivisto \& Mota 2007a) the authors use data from solar system, type Ia supernova, cosmic background radiation, large-scale structure and nucleosynthesis. They found some tension with nucleosynthesis and the baryon acoustic scale. However, their results are based on working in the special case of an exponential potential $V(\phi)$ for the scalar field. The intermediate evolution exists for a more elaborate scalar field potential, and this fact makes a study of this type of evolution imperative.

To test the model against the observation, it is useful to write down all the formulae in terms of the redshift, $z=-1+a\left(t_{0}\right) / a(t)$. We also impose that $a\left(t_{0}\right)=1$, given the relation

$\ln b(1+z)=-A t^{f}$.

From this equation we can rewrite the deceleration parameter (3) as

$q(z)=-1+\frac{f-1}{f} \frac{1}{\ln b(1+z)}$,

and the Hubble function can be written as

$H(z)=H_{0}\left[1+\frac{\ln (1+z)}{\ln b}\right]^{\frac{f-1}{f}}$.

Usually the ratio $E(z)=H(z) / H_{0}$ is what we need to perform the statistical analysis. It is clear that the free parameters of the model are: $h, f$ and $b$. The original parameter $A$ in (1) is related to $b$ through the age of the universe $t_{0}$, by $\ln b=-A t_{0}^{f}$.

Because this model shows a transition from a decelerated phase to an accelerated one, we choose to replace the $b$ parameter by the cross redshift $z_{c}$, at which $q=0$. From (5) we get

$\ln b=\frac{f-1}{f}-\ln \left(1+z_{c}\right)$.

In the next section we shall use (6), written in terms of the free parameters $h, f$ and $z_{c}$, to test the model against SNIa data, using both the Lick Observatory Supernova Search (LOSS) compilation set Ganeshalingam 2013) comprised of 586 SNIa, and the Joint Ligh-curve Analysis (JLA) set (Betoule et al. 2014) with 740 points, the largest compilation so far.

We also add baryon acoustic oscillation (BAO) data points from (Beutler et al. 2011), using the approach considered by Xia et al. (2012) and Dolgov et al. (2014). From the quoted references it is found that:

$d(z)=(0.335 \pm 0.016,0.576 \pm 0.022,1.539 \pm 0.039)$,

for $z=(0.106,0.2,0.57)$ respectively, where $d(z) \equiv$ $D_{V}(z) / D_{V}(z=0.35)$ with

$D_{V}(z)=\left[(1+z)^{2} D_{A}^{2}(z) \frac{c z}{H(z)}\right]^{1 / 3}$,

where $D_{A}(z)$ is the angular diameter distance defined by

$D_{A}(z)=\frac{c}{H_{0}(1+z)} \int_{0}^{z} \frac{d z^{\prime}}{E\left(z^{\prime}\right)}$,

valid for flat space.

\section{Testing the model}

In this section we use the Loss compilation set Ganeshalingam 2013) and the JLA set (Betoule et al. 2014), together with the BAO points to constrain the free parameters for the intermediate evolution model.

In the case of the Loss compiled set Ganeshalingam 2013), the fitting is done by minimizing the $\chi^{2}$ function,

$\chi^{2}=\sum_{i} \frac{\left(\mu_{i}-\mu_{t h}\left(z_{i}\right)\right)^{2}}{\sigma_{i}^{2}}$,

where $\mu_{i}, \sigma_{i}$ are the observational values of the distance modulus and its errors, while $\mu_{t h}\left(z_{i}\right)$ is the theoretical value of the distance modulus evaluated at the observed redshift $z_{i}$

$\mu_{t h}(z)=m-M=5 \log _{10} D_{L}(z)+25$,

where

$D_{L}(z)=\frac{c}{H_{0}}(1+z) \int_{0}^{z} \frac{d z^{\prime}}{E\left(z^{\prime}\right)}$,

is the luminosity distance in flat space.

In the case of the JLA set (Betoule et al. 2014), the function we minimize is

$\chi^{2}=\left(\mu-\mu_{t h}\right)^{T} C^{-1}\left(\mu-\mu_{t h}\right)$,

where $C$ corresponds to the covariance matrix released in (Betoule et al. 2014), and the distance modulus is assumed to take the form

$\mu=m-M+\alpha X-\gamma Y$, 
where $m$ is the maximum apparent magnitude in the rest frame of the $\mathrm{B}$ band, $X$ is related to the time stretching of the light-curves, and $Y$ corrects the color at maximum brightness. In the general case, cosmology is restricted together with the parameters $M, X$ and $Y$. In (Betoule et al. 2014) is also given a compressed form of the data, where only $M$ is left as a free parameter.

\section{$3.1 \Lambda \mathrm{CDM}$ model}

In order to have a reference model to compare with, we start studying the performance of the $\Lambda$ CDM model against the JLA SNIa data set (Betoule et al. 2014). We assume a $\Lambda$ CDM model with arbitrary curvature to ensure three free parameters in the fitting process; $h$, $\Omega_{m}$ and $\Omega_{\Lambda}$. The Hubble parameter for this model is

$E^{2}(z)=\Omega_{m}(1+z)^{2} z-\Omega_{\Lambda} z(2+z)+(1+z)^{2}$.

The best fit gives $\chi_{\text {min }}^{2}=32.76$. After marginalizing in $h$, the following best fit parameters are obtained: $\Omega_{m}=0.19 \pm 0.11, \Omega_{\Lambda}=0.56 \pm 0.17$, which agree with the values reported in (Betoule et al. 2014) and in particular with Figure 15 in that paper.

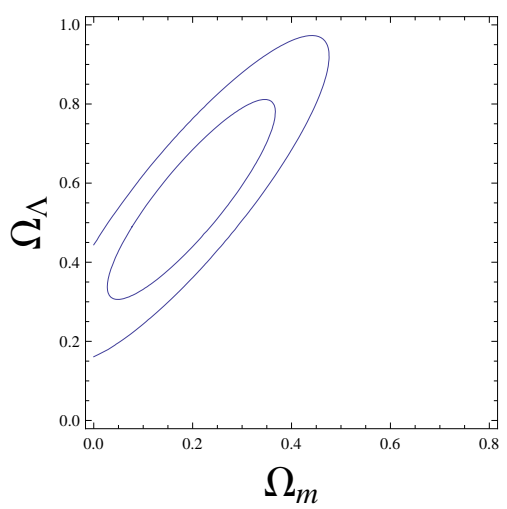

Fig. 1 We plot the contour at $1 \sigma$ and $2 \sigma$ for the parameters $\Omega_{m}$ and $\Omega_{\Lambda}$ after marginalization of $h$. Here we have used the compressed data from (Betoule et al. 2014).

\subsection{Intermediate model}

In order to compare with the results of the paper by Dolgov et al. (2014), here we describe the results using both SNIa data sets, together the BAO points.

The best fit values of the parameters - using the Loss set together with the BAO points - are: $f=0.48 \pm 0.06$, $z_{c}=0.63 \pm 0.07$ and $h=0.675 \pm 0.004$. Actually, these values remains essentially the same with and without taking the three BAO points into consideration. This fact is also mentioned by the authors of (Dolgov et al.

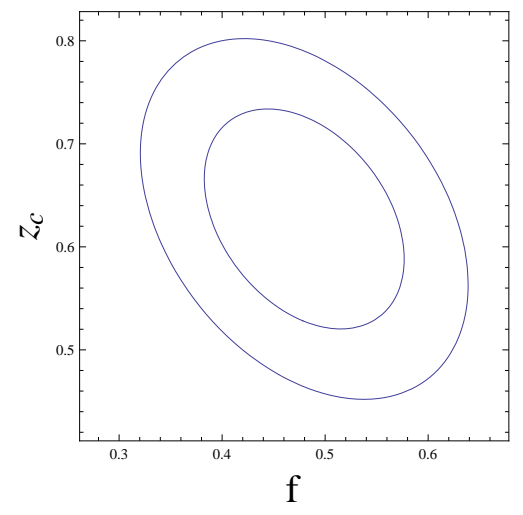

Fig. 2 We plot the contour at $1 \sigma$ and $2 \sigma$ for the parameters $f$ and $z_{c}$ after marginalization of $h$. Here we have used data from the Loss compilation set (Ganeshalingam 2013) and the BAO points discussed in the text.

2014) in the analysis they performed. After marginalization of the parameter $h$, we plot the confidence limits of the parameters $f$ and $z_{c}$ in Fig, 2 .

We follow Dolgov et al. (2014) and use the compressed form of the JLA likelihood where only $M$ is taken as a free parameter that is constrained together with the cosmological parameters. This procedure is equivalent to what we already did in the previous case using the Loss compiled data set. In that case, we consider $h$ as a free parameter, and after the analysis was performed, it was marginalized. This is the well-know degeneracy between the absolute magnitude and the Hubble constant.

Using the JLA data set (Betoule et al. 2014) alone, the best fit parameters are: $f=0.50 \pm 0.28, z_{c}=$ $1.2 \pm 1.3$ and $M=43.168 \pm 0.024$. Notice the large uncertainty in the determination of the cross redshift $z_{c}$. After marginalization of the nuisance parameter $M$, the confidence contour of the parameters $f$ and $z_{c}$ are displayed in Fig.(3).

After adding the BAO points, the result gives small changes compared with the previous case with SNIa alone, with the following best-fit parameters: $f=$ $0.52 \pm 0.21, z_{c}=0.93 \pm 0.71$ and $M=43.175 \pm 0.024$. After marginalization of the nuisance parameter $M$ we get the confidence contours for the parameters $f$ and $z_{c}$ displayed in Fig.(44). It is clear that adding the BAO points enables us to increase slightly the precision in the determination of the free parameters.

A final comment would be useful here. A direct comparison between Fig.(2) and Fig.(4) is not completely fair. In fact, the LOSS sample we have used, does not include the estimated systematic errors, whereas the JLA set does. This is the reason for the difference between the precision in the parameter determination observed in the graphs. 


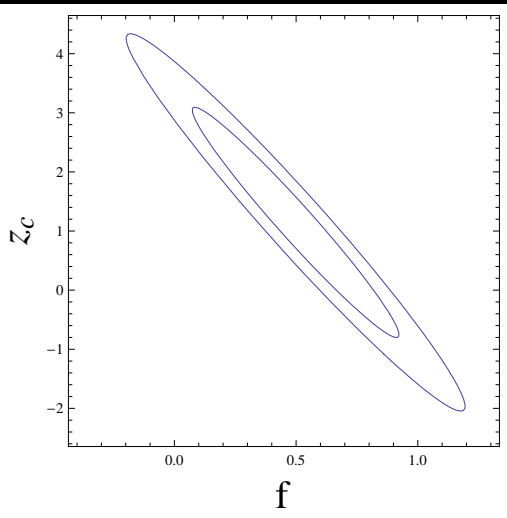

Fig. 3 We plot the contour at $1 \sigma$ and $2 \sigma$ for the parameters $f$ and $z_{c}$ after marginalization of $M$. Here we have used data only from the JLA set (Betoule et al. 2014).

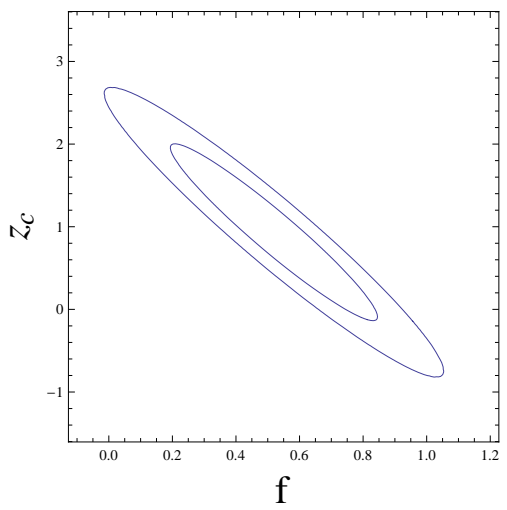

Fig. 4 We plot the contour at $1 \sigma$ and $2 \sigma$ for the parameters $f$ and $z_{c}$ after marginalization of $M$. Here we have used SNIa data from the JLA (Betoule et al. 2014) and the BAO points.

\section{Discussion}

From a theoretical point of view, we know that the intermediate evolution is more appropriated to describe the low redshift evolution, because this allows the existence of a transition from the decelerated expansion phase to the current accelerated expansion phase.

In previous sections, we showed that the intermediate evolution successfully fit the data from SNIa and BAO. In fact, for the best fit using SNIa (JLA set) only, we get $\chi_{\min }^{2}=32.89$ for the intermediate evolution (with three free parameters, $f, z_{c}$ and $M$ ), whereas within the $\Lambda \mathrm{CDM}$ (with also three free parameters $\left(\Omega_{m}\right.$, $\Omega_{\Lambda}$ and $\left.h\right)$ ) we get $\chi_{\min }^{2}=32.76$, both certainly a good fit to the data (for the binned JLA set with 31 points, the estimated variance in $\chi^{2}$ is $\sigma \simeq \sqrt{2 / 31} \simeq 0.25$, which means that both fit are comparable to each other (see Andrae et al. (2010))).

Just to make the comparison more evident, we plot the binned data from the JLA set (Betoule et al. 2014), with the best fit curve obtained from the theoretical model we studied - the intermediate evolution Eq.(1) - and the power-law model $a(t) \simeq t^{\beta}$ with $\beta=1.55$ (quoted from (Dolgov et al. 2014)) in Fig.(15).

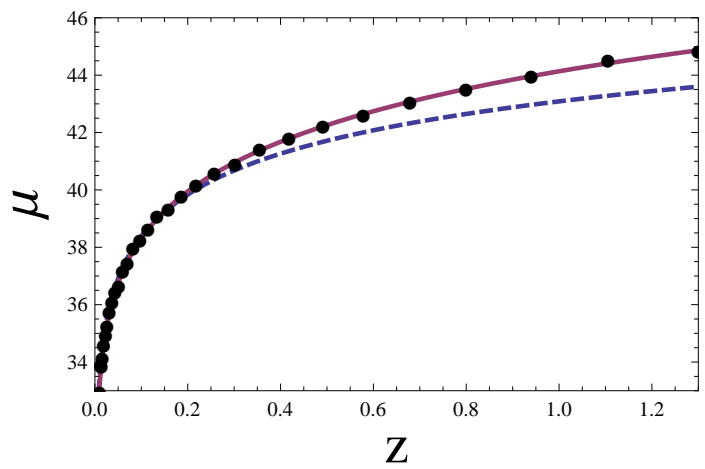

Fig. 5 We plot the 31 data points from the JLA set (Betoule et al. 2014) together with the best-fit value found in this work, using the intermediate evolution (11) (continuous line), and the best fit found in (Dolgov et al. 2014) using a power-law evolution $a(t) \simeq t^{\beta}$ with $\beta=1.55$ quoted from that paper (dashed line). Notice how the power-law fit moves away the data points from $z>0.2$.

It is clear that the intermediate evolution provides a better fit to the data, than the power-law with $\beta=1.55$.

Actually, our study suggests that if we only use SNIa data, using all the data from the LOSS compiled set, and independently using all the data from the compressed JLA set, the power-law best fit does not correspond to $\beta \simeq 3 / 2$ rather we obtain $\beta=1.78 \pm 0.09$ with LOSS, whereas $\beta=-0.35 \pm 0.06$ with JLA.

In this paper we have studied the intermediate evolution as the most appropriate for describing the low redshift regime supported by observations from type Ia supernovae and BAO. We found that the recent data (using the LOSS compiled sample (Ganeshalingam 2013) and JLA set (Betoule et al. 2014)) suggest that the intermediate evolution characterized by the scale factor (11) is as good a fit to this redshift regime as the standard $\Lambda$ CDM model.

This result can be considered a step forward from (Dolgov et al. 2014), where the authors used a powerlaw type evolution $a(t) \simeq t^{\beta}$ and studied the constraints the data imposed on the parameter $\beta$. Although they successfully produce a good fit to the SNIa data, the model they consider is unable to describe a transition from a decelerated to an accelerated expansion phase. This transition is a key ingredient that any model must satisfy if it claims to describe the recent (low redshift $z<1$ ) evolution of the universe, which is essentially the same redshift range as the supernova data span.

Although these results seems to rule out the powerlaw evolution to describe the low redshift regime (unless 
we are interested in fitting the very low regime $z<0.2$ ), the original motivation, that of confronting modified gravity theories to data looking for signals in favor of a dynamic adjustment mechanism to the vacuum energy problem, remains intact. Actually, this becomes even more interesting with the connection with the GaussBonnet coupling terms needed in the action to get the intermediate evolution.

\section{Acknowledgements}

VHC acknowledges financial support through DIUV 50/2013 and FONDECYT 1110230. This work is dedicated to the memory of our friend and colleague Sergio del Campo with whom this project was initiated in July 2014. 


\section{References}

Anderson, L., et al., 2012, Mon. Not. R. Astron. Soc.427, 3435

Andrae, R., Schulze-Hartung, T., Melchior, P., 2010, preprint arXiv:1012.3754

Antoniadis, I., Rizos, J., Tamvakis, K., 1994, Nucl. Phys. B 415, 497

Barrow, J.D., Phys. Lett. B 235, 40 (1990);

Barrow, J.D., Liddle, A.R., Pahud, C., 2006, Phys. Rev. D74, 127305

Barrow, J.D., Saich, P., 1990, Phys. Lett. B 249, 406

Betoule, M., et al. [SDSS Collaboration], 2014, Astron. Astrophys.568, A22

Beutler, F., et al., 2011, Mon. Not. R. Astron. Soc.416, 3017

Boulware, D.G., Deser, S., 1985, Phys. Rev. Lett.55, 2656

Boulware, D.G., Deser, S., 1986, Phys. Lett. B 175, 409

Cognola, G., Elizalde, E., Nojiri, S., Odintsov, S.D., Zerbini, S., 2006, Phys. Rev. D73, 084007

Chen, Ch.-M., Galtsov, D.V., Orlov, D.G., 2007, Phys. Rev. D75, 084030

de la Cruz-Dombriz, A., Saez-Gomez, D., 2012, Class. Quant. Grav. 29, 245014

del Campo, S., 2014, preprint arXiv:1404.1649 [astroph.CO].

De Felice, A., Mota, D.F., Tsujikawa, S., 2010, Phys. Rev. D81, 023532

Dolgov, A.D., 1997, Phys. Rev. D55, 5881

Dolgov, A., Halenka, V., Tkachev, I., 2014, J. Cosmol. Astropart. Phys.10 047

Frieman, J., Turner, M., Huterer, D., 2008, Annu. Rev. Astron. Astrophys., 46, 385, 2008

Ganeshalingam, M., Li, W., Filippenko, A.V., 2013, Mon. Not. R. Astron. Soc.433, 2240

Granda, L.N., Jimenez, D.F., 2014, Phys. Rev. D90, no. 12, 123512

Hebecker, A., Wetterich, C., 2000, Phys. Rev. Lett.85, 3339

Kanti, P., Mavromatos, N.E., Rizos, J., Tamvakis, K., Winstanley, E., 1996, Phys. Rev. D54 5049

Koivisto, T., Mota, D., 2007, Phys. Lett. B 644, 104

Koivisto, T., Mota, D., Phys. Rev. D75, 023518

Martin, J., 2008, Mod. Phys. Lett. A 23, 1252

Mignemi, S., Stewart, N.R., 1993, Phys. Rev. D47, 5259

Muslimov, A., 1990, Class. Quantum Grav. 7, 231

Nojiri, S., Odintsov, S.D., 2005, Phys. Lett. B 631, 1

Nojiri, S., Odintsov, S.D., Sasaki, M., 2005, Phys. Rev. D71, 123509

Padmanabhan, T., 2003, Phys. Rep.380, 235

Padmanabhan, N., et al., 2012, Mon. Not. R. Astron. Soc.427, 2132

Peebles, P.J.E., Ratra,B., 2003, Rev. Mod. Phys. 75, 559

Perivolaropoulos, L., 2010, J. Phys.: Conf. Ser. 222, 1, 012024

Perlmutter, S. et al., 1999, Astrophys. J.517, 565

Riess, A.G. et al., 1998, Astron. J.116, 1009

Sanyal, A.K., 2007, Phys. Lett. B 6451

Sanyal, A.K., 2009, Gen. Rel. Grav. 41, 1511

Suzuki, N., Rubin, D., Lidman, C., et al. 2012, Astrophys. J., 746, 85

Tsujikawa, S., 2010, Lect. Notes Phys. 800, 99

Weinberg, D.H. et al., 2013, Phys. Rep.530, 2, 87-255

Weinberg, S., 1989, Rev. Mod. Phys. 61, 1 (1989);

Xia, J.Q., Vitagliano, V., Liberati, S., Viel, M., 2012, Phys. Rev. D85, 043520

This manuscript was prepared with the AAS LATEX macros v5.2. 